\title{
RINGS OF INVARIANT POLYNOMIALS FOR A CLASS OF LIE ALGEBRAS $\left({ }^{1}\right)$
}

\author{
BY
}

\author{
S. J. TAKIFF
}

\begin{abstract}
Let $G$ be a group and let $\pi: G \rightarrow G L(V)$ be a finite-dimensional representation of $G$. Then for $g \in G, \pi(g)$ induces an automorphism of the symmetric algebra $S(V)$ of $V$. We let $I(G, V, \pi)$ be the subring of $S(V)$ consisting of elements invariant under this induced action. If $G$ is a connected complex semisimple Lie group with Lie algebra $L$ and if Ad is the adjoint representation of $G$ on $L$, then Chevalley has shown that $I(G, L, A d)$ is generated by a finite set of algebraically independent elements. However, relatively little is known for nonsemisimple Lie groups. In this paper the author exhibits and investigates a class of nonsemisimple Lie groups $G$ with Lie algebra $L$ for which $I(G, L, A d)$ is also generated by a finite set of algebraically independent elements.
\end{abstract}

1. Let $G$ be a group, let $V$ be a finite-dimensional vector space over a field $F$ with basis $\left\{v_{1}, \ldots, v_{m}\right\}$, and let $\pi$ be a representation of $G$ on $V, \pi: G \rightarrow G L(V)$. Then for $g \in G, \pi(g)$ induces an automorphism, also denoted by $\pi(g)$, on the symmetric algebra of $V, S(V)=F\left[v_{1}, \ldots, v_{m}\right]$. We say that $p\left(v_{1}, \ldots, v_{m}\right) \in S(V)$ is an invariant polynomial for $(G, V, \pi)$ if

$$
\pi(g) p\left(v_{1}, \ldots, v_{m}\right)=p\left(\pi(g) v_{1}, \ldots, \pi(g) v_{m}\right)=p\left(v_{1}, \ldots, v_{m}\right),
$$

for all $g \in G$. Let $I(G, V, \pi)$ be the algebra of all invariant polynomials for $(G, V, \pi)$. $I(G, V, \pi)$ is clearly independent of the choice of the basis $\left\{v_{1}, \ldots, v_{m}\right\}$ for $V$.

More specifically, let $G$ be a connected complex semisimple Lie group with Lie algebra $L$, and let Ad be the adjoint representation of $G$ on $L$. Then $I(G, L, A d)$ is generated by $l$ algebraically independent homogeneous polynomials, where $l$ equals the rank of $L$. This theorem is due to Chevalley, see [1, Theorem A, p. 778] and [5, Theorem 5.37, p. 507]. Another example that should be mentioned is as follows. Let $G=R^{4}$ (S $S O(1,3)$ be the inhomogeneous Lorentz group, $R$ being the field of real numbers, then $I\left(R^{4} \subseteq S O(1,3), R^{4} \oplus \mathfrak{s} \mathfrak{p}(1,3)\right.$, Ad) is generated by 2 algebraically independent homogeneous polynomials of degrees 2 and 4 . This

Received by the editors October 27, 1970.

AMS 1970 subject classifications. Primary 22E99; Secondary 15A72, 17B35.

Key words and phrases. Symmetric algebra, ring of invariant polynomials, adjoint representation, polynomial functions, contragredient representation, algebraically independent homogeneous polynomials, Levi decomposition, principal nilpotent element, differential operators, formal power series.

( $\left.{ }^{1}\right)$ This paper is a portion of the author's doctoral dissertation which was completed at the University of Illinois in 1969 under the direction of Professor R. Ranga Rao. The author wishes to express his gratitude to Professor Rao for his encouragement and guidance.

Copyright (C) 1971, American Mathematical Society 
result was originally proved several years ago by V. S. Varadarajan during a series of lectures at the Indian Statistical Institute at Calcutta.

Now besides the result for $R^{4}$ (S $S O(1,3)$, little is known about $I(G, L, \mathrm{Ad})$ for Lie groups which are not semisimple. It is the purpose of this paper to exhibit a class of complex Lie algebras,

$\left\{\Omega^{h}(L) \mid L\right.$ is any complex semisimple Lie algebra $\}$,

with the following properties: If $G\left(\Omega^{h}(L)\right)$ is any connected Lie group with Lie algebra $\Omega^{h}(L)$, then $I\left(G\left(\Omega^{h}(L)\right), \Omega^{h}(L)\right.$, Ad) is generated by $\left(2^{h}\right) l$ algebraically independent homogeneous polynomials, where $l$ equals the rank of $L$. Further, $\Omega^{h}(L)=\operatorname{Rad}\left(\Omega^{h}(L)\right) \oplus L$ is a Levi decomposition, where the radical of $\Omega^{h}(L)$, $\operatorname{Rad}\left(\Omega^{h}(L)\right)$, is nilpotent and has a lower central series of length $h$.

The author would like to acknowledge the paper of V. S. Varadarajan [6] for some important techniques used in this paper.

2. Two useful tools must be presented before we proceed. First, let $(G, V, \pi)$ be as above, and let $V^{*}$ be the dual space of $V$. Then the algebra of $F$-valued polynomial functions on $V, P(V)$, is equal to $S\left(V^{*}\right)$. We shall say that $p(v) \in P(V)$ is an invariant polynomial function for $(G, V, \pi)$ if

$$
\pi(g) p(v)=p(\pi(g) v)=p(v) \text { for all } v \in V \text { and } g \in G .
$$

We let $I F(G, V, \pi)$ denote the algebra of all invariant polynomial functions for $(G, V, \pi)$. Now if $V^{* *}$ is the dual space of $V^{*}$, then there is a natural isomorphism between $S(V)$ and $P\left(V^{*}\right)$. So let $\pi^{*}$ be the representation of $G$ on $V^{*}$ contragredient to $\pi$; that is, $\pi^{*}(g) v^{*}(w)=v^{*}\left(\pi\left(g^{-1}\right) w\right), w \in V, v^{*} \in V^{*}, g \in G$. Then the above isomorphism between $S(V)$ and $P\left(V^{*}\right)$ induces an isomorphism between $I(G, V, \pi)$ and $\operatorname{IF}\left(G, V^{*}, \pi^{*}\right)$.

Next, let $G$ be a connected Lie group with Lie algebra $L$, having a basis $\left\{x_{1}, \ldots, x_{n}\right\}$. And let $\pi$ be an analytic representation of $G$ on a real vector space $V$. Then the differential $d \pi_{(1)}$ of $\pi$ evaluated at 1 , the identity of $G$, is a linear map of $L$ into the algebra of all linear transformations on $V$, hence $d \pi_{(1)}(x)$ extends to an algebra homomorphism of $S(V)$ into itself, $x \in L$. We therefore have for $p \in S(V)$

$$
\begin{aligned}
& p \in I(G, V, \pi) \\
& \text { if and only if } d \pi_{(1)}(x) p=(d / d t)\{\pi(\exp t x) p\}_{t=0}=0, \quad \text { for all } x \in L, \\
& \text { if and only if } d \pi_{(1)}\left(x_{i}\right) p=(d / d t)\left\{\pi\left(\exp t x_{i}\right) p\right\}_{t=0}=0, \quad i=1, \ldots, L, \\
& \text { if and only if } \pi\left(\exp t x_{i}\right) p=p, \quad \text { for all } t \in R, i=1, \ldots, n \text {. }
\end{aligned}
$$

We shall always let $t$ denote a real variable.

3. Suppose $L$ is a finite-dimensional Lie algebra with Lie product [, $]_{L}$ over a field $F, F=\boldsymbol{R}$ or $\boldsymbol{C}, \boldsymbol{C}$ being the field of complex numbers. Form the vector space direct sum $L \oplus L$, and write the elements of $L \oplus L$ as ordered pairs $\left(l_{1}, l_{2}\right)$, $l_{1}, l_{2} \in L$. Then we define the following product:

$$
\left[\left(l_{1}, l_{2}\right),\left(l_{1}^{\prime}, l_{2}^{\prime}\right)\right]=\left(\left[l_{1}, l_{2}^{\prime}\right]_{L}+\left[l_{2}, l_{1}^{\prime}\right]_{L},\left[l_{2}, l_{2}^{\prime}\right]_{L}\right),
$$


where $l_{1}, l_{2}, l_{1}^{\prime}, l_{2}^{\prime} \in L$. Under this product $L \oplus L$ becomes a Lie algebra, see [2, pp. 16-18], and we shall denote it by $\Omega(L)$.

For the remainder of the paper we shall drop the " $L$ " from $[,]_{L}$.

Now let $\bar{L}=\Omega(L)$, let $\bar{G}$ be a connected Lie group whose Lie algebra is $\bar{L}$ and let $G$ be a connected Lie subgroup of $\bar{G}$ whose Lie algebra is $L$. Then if Ad is the adjoint representation of $\bar{G}$ on $\bar{L}$, a simple computation shows that

$\operatorname{Ad}\left(\exp \left(u_{1}, u_{2}\right)\right)\left(l_{1}, l_{2}\right)$

$$
=\left(\left[u_{1}, \operatorname{Ad}\left(\exp u_{2}\right) l_{2}\right]+\operatorname{Ad}\left(\exp u_{2}\right) l_{1}, \operatorname{Ad}\left(\exp u_{2}\right) l_{2}\right), \quad u_{1}, u_{2}, l_{1}, l_{2} \in L .
$$

Now let $\left\{x_{1}, \ldots, x_{n}\right\}$ be a basis for $L$; then $\left\{\left(x_{1}, 0\right), \ldots,\left(x_{n}, 0\right),\left(0, x_{1}\right), \ldots,\left(0, x_{n}\right)\right\}$ is a basis for $\bar{L}$. Finally, we note from $\S 2$ that $p \in I(\bar{G}, \bar{L}, \mathrm{Ad})$ if and only if $\operatorname{Ad}(\exp (0, u)) p=p$ and $\operatorname{Ad}(\exp (u, 0)) p=p$, for all $u \in L, p \in S(\bar{G})$.

4. Now let $X_{1}, \ldots, X_{n}, Y_{1}, \ldots, Y_{n}, Z_{1}, \ldots, Z_{n}$ denote indeterminates over $F$ and let $p\left(X_{1}, \ldots, X_{n}\right)$ be a homogeneous polynomial. Then we have

$$
\begin{aligned}
p\left(X_{1}+t Y_{1}, \ldots, X_{n}+t Y_{n}\right)= & p\left(X_{1}, \ldots, X_{n}\right)+t q\left(X_{1}, \ldots, X_{n}, Y_{1}, \ldots, Y_{n}\right) \\
& +\sum_{m \geqq 2} t^{m} h_{m}\left(X_{1}, \ldots, X_{n}, Y_{1}, \ldots, Y_{n}\right)
\end{aligned}
$$

where $q$ and the $h_{m}$ are also homogeneous polynomials. Further, we have

$$
\begin{aligned}
p\left(X_{1}+t Y_{1}+t^{2} Z_{1}, \ldots, X_{n}+t Y_{n}+t^{2} Z_{n}\right) & \\
= & p\left(X_{1}, \ldots, X_{n}\right)+t q\left(X_{1}, \ldots, X_{n}, Y_{1}, \ldots, Y_{n}\right) \\
& +\sum_{m \geqq 2} t^{m} k_{m}\left(X_{1}, \ldots, X_{n}, Y_{1}, \ldots, Y_{n}, Z_{1}, \ldots, Z_{n}\right),
\end{aligned}
$$

where the $k_{m}$ are homogeneous polynomials. Finally we note that

$$
q\left(X_{1}, \ldots, X_{n}, Y_{1}, \ldots, Y_{n}\right)=\sum_{i=1}^{n} D_{i} p\left(X_{1}, \ldots, X_{n}\right) Y_{i}
$$

where $D_{i}$ is the unique $F$-derivation on $F\left[X_{1}, \ldots, X_{n}\right]$ such that $D_{i}\left(X_{j}\right)=\delta_{i j}, i$, $j=1, \ldots, n\left(\delta_{i j}=1\right.$ if $i=j$ and $\delta_{i j}=0$ if $\left.i \neq j\right)$. Hence, if $p\left(X_{1}, X_{2}, X_{3}\right)=X_{1} X_{2} X_{3}+X_{1}^{3}$, then

$$
q\left(X_{1}, X_{2}, X_{3}, Y_{1}, Y_{2}, Y_{3}\right)=X_{2} X_{3} Y_{1}+X_{1} X_{3} Y_{2}+X_{1} X_{2} Y_{3}+3 X_{1}^{2} Y_{1}
$$

THEOREM 4.1. If $p\left(X_{1}, \ldots, X_{n}\right)$ is a homogeneous polynomial such that $p\left(x_{1}, \ldots, x_{n}\right) \in I(G, L, \mathrm{Ad})$, then

1. $p\left(\left(x_{1}, 0\right), \ldots,\left(x_{n}, 0\right)\right) \in I(\bar{G}, \bar{L}, \mathrm{Ad})$, and

2. $q\left(\left(x_{1}, 0\right), \ldots,\left(x_{n}, 0\right),\left(0, x_{1}\right), \ldots,\left(0, x_{n}\right)\right) \in I(\bar{G}, \bar{L}$, Ad $)$, where $q\left(X_{1}, \ldots, X_{n}\right)$ $=\sum D_{i} p\left(X_{1}, \ldots, X_{n}\right) Y_{i}$.

Proof. It is clear from the last paragraph of $\S 3$ that $\operatorname{Ad}(\exp (0, u)) p\left(\left(x_{1}, 0\right), \ldots,\left(x_{n}, 0\right)\right)=p\left(\left(x_{1}, 0\right), \ldots,\left(x_{n}, 0\right)\right)$ for all $u \in L$. 
Further, since $\operatorname{Ad}(\exp (u, 0))(x, 0)=(x, 0)$ for all $x, u \in L$ then we also have

$$
\operatorname{Ad}(\exp (u, 0)) p\left(\left(x_{1}, 0\right), \ldots,\left(x_{n}, 0\right)\right)=p\left(\left(x_{1}, 0\right), \ldots,\left(x_{n}, 0\right)\right) \text {. }
$$

Therefore $p\left(\left(x_{1}, 0\right), \ldots,\left(x_{n}, 0\right)\right) \in I(\bar{G}, \bar{L}$, Ad $)$.

Now let $u \in L$ and assume that $\operatorname{Ad}(\exp (0, u))\left(x_{i}, 0\right)=\sum_{j=1}^{n} c_{i j}\left(x_{j}, 0\right)$, where $c_{i j} \in F, i, j=1, \ldots, n$. Then also

$$
\operatorname{Ad}(\exp (0, u))\left(\left(x_{i}, 0\right)+t\left(0, x_{i}\right)\right)=\sum_{j=1}^{n} c_{i j}\left(\left(x_{j}, 0\right)+t\left(0, x_{j}\right)\right), \quad i=1, \ldots, n .
$$

Hence,

$$
\begin{aligned}
\operatorname{Ad}(\exp (0, u)) p\left(\left(x_{1}, 0\right)+\right. & \left.t\left(0, x_{1}\right), \ldots,\left(x_{n}, 0\right)+t\left(0, x_{n}\right)\right) \\
& =p\left(\left(x_{1}, 0\right)+t\left(0, x_{1}\right), \ldots,\left(x_{n}, 0\right)+t\left(0, x_{n}\right)\right) \text { for all } u \in L .
\end{aligned}
$$

Therefore, from the first paragraph of this section,

$\operatorname{Ad}(\exp (0, u)) q\left(\left(x_{1}, 0\right), \ldots,\left(x_{n}, 0\right),\left(0, x_{1}\right), \ldots,\left(0, x_{n}\right)\right)$

$$
=q\left(\left(x_{1}, 0\right), \ldots,\left(x_{n}, 0\right),\left(0, x_{1}\right), \ldots,\left(0, x_{n}\right)\right) \text { for all } u \in L .
$$

We must now show that $q\left(\left(x_{1}, 0\right), \ldots,\left(x_{n}, 0\right),\left(0, x_{1}\right), \ldots,\left(0, x_{n}\right)\right)$ is invariant under $\operatorname{Ad}(\exp (u, 0))$ for all $u \in L$. First we observe that

$$
\begin{aligned}
p\left(\left(x_{1}, 0\right), \ldots,\right. & \left.\left(x_{n}, 0\right)\right) \\
= & \operatorname{Ad}(\exp t(0, u)) p\left(\left(x_{1}, 0\right), \ldots,\left(x_{n}, 0\right)\right) \\
= & p\left(\left(x_{1}, 0\right)+t\left(\left[u, x_{1}\right], 0\right)+t^{2}\left(v_{1}, 0\right), \ldots,\left(x_{n}, 0\right)+t\left(\left[u, x_{n}\right], 0\right)+t^{2}\left(v_{n}, 0\right)\right) \\
= & p\left(\left(x_{1}, 0\right), \ldots,\left(x_{n}, 0\right)\right) \\
& \quad+t q\left(\left(x_{1}, 0\right) \ldots,\left(x_{n}, 0\right),\left(\left[u, x_{1}\right], 0\right), \ldots,\left(\left[u, x_{n}\right], 0\right)\right)+t^{2} w,
\end{aligned}
$$

where $u \in L$ and $w$ and the $v_{i}$ are power series in $t$ with coefficients in $S(L)$. Hence,

$$
q\left(\left(x_{1}, 0\right), \ldots,\left(x_{n}, 0\right),\left(\left[u, x_{1}\right], 0\right), \ldots,\left(\left[u, x_{n}\right], 0\right)\right)=0 .
$$

Therefore,

$\operatorname{Ad}(\exp t(u, 0)) q\left(\left(x_{1}, 0\right), \ldots,\left(x_{n}, 0\right),\left(0, x_{1}\right), \ldots,\left(0, x_{n}\right)\right)$

$$
\begin{aligned}
= & q\left(\left(x_{1}, 0\right), \ldots,\left(x_{n}, 0\right),\left(0, x_{1}\right)+t\left(\left[u, x_{1}\right], 0\right), \ldots,\left(0, x_{n}\right)+t\left(\left[u, x_{n}\right], 0\right)\right) \\
= & q\left(\left(x_{1}, 0\right), \ldots,\left(x_{n}, 0\right),\left(0, x_{1}\right), \ldots,\left(0, x_{n}\right)\right) \\
& \quad+t q\left(\left(x_{1}, 0\right), \ldots,\left(x_{n}, 0\right),\left(\left[u, x_{1}\right], 0\right), \ldots,\left(\left[u, x_{1}\right], 0\right)\right) \\
= & q\left(\left(x_{1}, 0\right), \ldots,\left(x_{n}, 0\right),\left(0, x_{n}\right), \ldots,\left(0, x_{n}\right)\right) \quad \text { for all } u \in L ;
\end{aligned}
$$

and so we are done.

Before proceeding to the next theorem we need the following

LEMMA 4.2. Let $K$ be any field of characteristic zero, and let $Y_{1}, \ldots, Y_{2 n}$ be algebraically independent over $K$. Set $A=K\left[Y_{1}, \ldots, Y_{n}\right] \subset B=K\left[Y_{1}, \ldots, Y_{2 n}\right]$ and denote the quotient field of $B$ by $(B)$. Let $E_{i}$ be the unique $K$-derivation of $B$ such that 
$E_{i}\left(Y_{j}\right)=\delta_{i j}, i, j=1, \ldots, 2 n$. If $p_{1}, \ldots, p_{r} \in A$ are algebraically independent over $K$, then $p_{1}, \ldots, p_{r}, \Delta p_{1}, \ldots, \Delta p_{r}$ are also algebraically independent over $K$, where

$$
\Delta p_{j}=\sum_{i=1}^{n} E_{i}\left(p_{j}\right) Y_{n+i}, \quad j=1, \ldots, r .
$$

Proof. If $q_{1}, \ldots, q_{m} \in B$, then it is well known that $q_{1}, \ldots, q_{m}$ are algebraically independent over $K$ if and only if $m$ equals the rank over $(B)$ of the matrix $\left(E_{i}\left(q_{j}\right)\right)_{j=1, \ldots, 2 n}^{i=1, \ldots, m}$. Therefore, since $p_{1}, \ldots, p_{r}$ are algebraically independent over $K$, we can assume for some $\lambda_{1}, \ldots, \lambda_{r}$ that the determinant of $M=\left(E_{\lambda_{i}}\left(p_{j}\right)\right)_{j=1, \ldots, r}^{i=1, \ldots, r}$ is not zero. Now, letting $p_{r+i}=\Delta p_{i}$ and $\lambda_{n+i}=n+\lambda_{i}, i=1, \ldots, r$, it is clear that the matrix

$$
\left(E_{\lambda_{1}}\left(p_{j}\right)\right)_{j=1, \ldots, 2 r}^{i=1, \ldots, r, n+1, \ldots, n+r}=\left(\begin{array}{l|l}
M & 0 \\
\hdashline & \frac{M}{*}
\end{array}\right) .
$$

Hence the determinant of this matrix is not zero, and so $2 r$ equals the rank of the matrix $\left(E_{i}\left(p_{j}\right)\right)_{j=1, \ldots, 2,2 r}^{i=1, \ldots, 2}$ This shows that $p_{1}, \ldots, p_{r}, \Delta p_{1}, \ldots, \Delta p_{r}$ are indeed algebraically independent over $K$.

TheOREM 4.3. Let $I(G, L$, Ad) have at least $b$ algebraically independent homogeneous polynomials of distinct degrees $d_{1}, \ldots, d_{s}$ and assume $n_{i}$ of them are of degree $d_{i}, i=1, \ldots, s$. Then $I(\bar{G}, \bar{L}$, Ad) has at least $2 b$ algebraically independent homogeneous polynomials and $2 n_{i}$ of these are of degree $d_{i}, i=1, \ldots, s$.

Proof. Let $p_{1}\left(x_{1}, \ldots, x_{n}\right), \ldots, p_{b}\left(x_{1}, \ldots, x_{n}\right)$ be algebraically independent homogeneous polynomials in $I(G, L, \mathrm{Ad})$. And let $p_{1}\left(X_{1}, \ldots, X_{n}\right), \ldots, p_{b}\left(X_{1}, \ldots, X_{n}\right)$ be the homogeneous polynomials in $F\left[X_{1}, \ldots, X_{n}\right]$ associated with these polynomials. Now define

$$
q,\left(X_{1}, \ldots, X_{n}, Y_{1}, \ldots, Y_{n}\right)=\sum_{i=1}^{n} D_{i} p_{j}\left(X_{1}, \ldots, X_{n}\right) Y_{i}, \quad j=1, \ldots, b,
$$

as in the first paragraph of this section. Then by Lemma $4.2, p_{1}\left(\left(x_{1}, 0\right), \ldots,\left(x_{n}, 0\right)\right)$, $\ldots, p_{b}\left(\left(x_{1}, 0\right), \ldots,\left(x_{n}, 0\right)\right), q_{1}\left(\left(x_{1}, 0\right), \ldots,\left(x_{n}, 0\right),\left(0, x_{1}\right), \ldots,\left(0, x_{n}\right)\right), \ldots$, $q_{b}\left(\left(x_{1}, 0\right), \ldots,\left(x_{n}, 0\right),\left(0, x_{1}\right), \ldots,\left(0, x_{n}\right)\right)$ are algebraically independent and by Theorem 4.1, they are homogeneous elements of $I(\bar{G}, \bar{L}, \mathrm{Ad})$.

5. Since in $\S \S 3$ and $4 L$ was an arbitrary finite-dimensional Lie algebra, it is clear that we can define $\Omega^{h}(L)=\Omega\left(\Omega^{h-1}(L)\right), h \geqq 1$, where $\Omega^{1}(L)=\Omega(L)$. So let $h$ be an arbitrary positive integer, let $\bar{L}=\Omega^{h}(L)$, and let $\bar{G}$ be a connected Lie group with Lie algebra $\bar{L}$.

THEOREM 5.1. Let $I(G, L$, Ad) have at least $b$ algebraically independent homogeneous polynomials of distinct degrees $d_{1}, \ldots, d_{s}$ and assume $n_{i}$ of them are of degree $d_{i}, i=1, \ldots, s$. Then $I(\bar{G}, \bar{L}, \mathrm{Ad})$ has at least $\left(2^{h}\right) b$ algebraically independent homogeneous polynomials and $\left(2^{h}\right) n_{i}$ of these are of degree $d_{i}, i=1, \ldots, s$. 
Proof. The proof is a direct consequence of the definition of $\Omega^{h}(L)$ and repeated use of Theorem 4.3.

6. We now study the Lie product operation in $\bar{L}=\Omega^{h}(L)$. Let $N=2^{h}$ and $M=2^{h-1}$, then as a vector space $\Omega^{h}(L)=L \oplus \cdots \oplus L$, the direct sum of $N$ copies of $L$. We will denote the elements of $\Omega^{h}(L)$ as $N$-tuples with coordinates in $L$; that is, $\bar{L}=\left\{\left(a_{1}, \ldots, a_{N}\right) \mid a_{i} \in L, i=1, \ldots, N\right\}$. (We omit intermediate parentheses; for example, $\left(\left(a_{1}, a_{2}\right),\left(a_{3}, a_{4}\right)\right)=\left(a_{1}, a_{2}, a_{3}, a_{4}\right)$ in $\Omega^{2}(L)$.) For convenience, we adopt the following notation:

$$
a e_{i, N}=\left(\begin{array}{c}
i \\
0, \ldots, 0, a, 0, \ldots, 0
\end{array}\right)
$$

where $a \in L$. Hence, $\left(a_{1}, a_{2}, \ldots, a_{N}\right)=\sum_{i=1}^{N} a_{i} e_{i, N}$ for $\left(a_{1}, a_{2}, \ldots, a_{N}\right) \in \bar{L}$, and $L e_{i, N}=\left\{a e_{i, N} \mid a \in L\right\}, i=1, \ldots, N$.

LEMMA 6.1. Let $a, b_{1}, \ldots, b_{N} \in L$, then

$$
\left[a e_{k, N}, \sum_{i=1}^{N} b_{i} e_{i, N}\right]=\sum_{i=1}^{k} c_{k, i}\left[a, b_{N-k+i}\right] e_{i, N}
$$

where $1 \leqq k \leqq N, c_{k, i}=0$ or 1 and $c_{k, 1}=1=c_{k, k}, k=1, \ldots, N, i=1, \ldots, k$.

Proof. By definition of the Lie product operation in $\Omega(L)$, the lemma is clearly true for $h=1$. So let us assume that it is true for $h=m$ and prove it true for $h=m+1$.

Case 1. $1 \leqq k \leqq M$. Then

$$
\begin{aligned}
{\left[a e_{k, N}, \sum_{i=1}^{N} b_{l} e_{i, N}\right] } & =\left[\left(a e_{k, M}, 0 e_{M, M}\right),\left(\sum_{i=1}^{M} b_{i} e_{i, M}, \sum_{i=1}^{M} b_{M+i} e_{i, M}\right)\right] \\
& =\left(\left[a e_{k, M}, \sum_{i=1}^{M} b_{M+i} e_{i, M}\right], 0 e_{M, M}\right) \\
& =\left(\sum_{i=1}^{k} d_{\kappa, i}\left[a, b_{N-k+i}\right] e_{i, M}, 0 e_{M, M}\right),
\end{aligned}
$$

where $d_{k, i}=0$ or $1, i=1, \ldots, k$, and $d_{k, 1}=1=d_{k, k}$. Furthermore, this last expression can be written as $\sum_{i=1}^{k} c_{k, i}\left[a, b_{N-k+i}\right] e_{i, N}$, where $c_{k, i}=d_{k, i}, i=1, \ldots, k$.

Case 2. $M<k \leqq N$. Then

$$
\begin{aligned}
{\left[a e_{k, N}, \sum_{i=1}^{N} b_{i} e_{i, N}\right] } & \\
= & {\left[\left(0 e_{M, M}, a e_{k-M, M}\right),\left(\sum_{i=1}^{M} b_{i} e_{i, M}, \sum_{i=1}^{M} b_{M+i} e_{i, M}\right)\right] } \\
& =\left(\left[a e_{k-M, M}, \sum_{i=1}^{M} b_{i} e_{i, M}\right],\left[a e_{k-M, M}, \sum_{i=1}^{M} b_{M+i} e_{i, M}\right]\right) \\
& =\left(\sum_{i=1}^{k-M} d_{k-M, i}\left[a, b_{M-(k-M)+i}\right] e_{i, M}, \sum_{i=1}^{k-M} f_{k-M, i}\left[a, b_{N-(k-M)+i}\right] e_{i, M}\right)
\end{aligned}
$$


(where $d_{k-M, i}, f_{k-M, i}=0$ or 1 for $i=1, \ldots, k-M$ and $d_{k-M, 1}=1=f_{k-M, k-M}$ )

$$
\begin{aligned}
& =\sum_{i=1}^{k-M} d_{k-M, i}\left[a, b_{N-k+i}\right] e_{i, N}+\sum_{i=M+1}^{(k-M)+M} f_{k-M, i-M}\left[a, b_{N-(k-M)+(i-M)}\right] e_{i, N} \\
& =\sum_{i=1}^{k} c_{k, i}\left[a, b_{N-k+i}\right] e_{i, N}
\end{aligned}
$$

where $c_{k, i}=d_{k-M, i}, i=1, \ldots, k-M, c_{k, i}=0, i=k-M+1, \ldots, M$ (if $k<N$ ), and finally $c_{k, i}=f_{k-M, i-M}, i=M+1, \ldots, k$.

LEMMA 6.2. Let $a, b_{1}, \ldots, b_{N} \in L$, then

$$
\left[a e_{N, N}, \sum_{i=1}^{N} b_{i} e_{i, N}\right]=\sum_{i=1}^{N}\left[a, b_{i}\right] e_{i, N} .
$$

Proof. Again, this lemma is clear for $h=1$. We assume it is true for $h=m$ and prove it true for $h=m+1$ by the following computation:

$$
\begin{aligned}
{\left[a e_{N, N}, \sum_{i=1}^{N} b_{i} e_{i, N}\right] } & =\left[\left(0 e_{M, M}, a e_{M, M}\right),\left(\sum_{i=1}^{M} b_{i} e_{i, M}, \sum_{i=1}^{M} b_{M+i} e_{i, M}\right)\right] \\
& =\left(\sum_{i=1}^{M}\left[a, b_{i}\right] e_{i, M}, \sum_{i=1}^{M}\left[a, b_{M+i}\right] e_{i, M}\right)=\sum_{i=1}^{N}\left[a, b_{i}\right] e_{i, N}
\end{aligned}
$$

7. We proceed with our study of $\bar{L}=\Omega^{h}(L), h \geqq 1$, assuming $L$ to be a real or complex finite-dimensional semisimple Lie algebra. First, recall that the lower central series of an arbitrary Lie algebra $H$ is

$$
H=Z^{0}(H) \supset Z^{1}(H) \supset \ldots \supset Z^{k}(H)=\left[H, Z^{k-1}(H)\right] \supset \cdots .
$$

Then $H$ is nilpotent if $Z^{k}(H)=0$ for some integer $k$, and we call Cen $(H)=k$ the length of the lower central series if $k$ is minimal. It is the goal of this section to obtain a Levi decomposition [2, p. 91] and to study the radical of $\bar{L}$.

THEOREM 7.1. Let $L$ be a real or complex finite-dimensional semisimple Lie algebra, $\bar{L}=\Omega^{h}(L), N=2^{h}$. Then $R=\sum_{i=1}^{N-1} L e_{i, N}$ is the radical of $\bar{L}$ and $L e_{N, N}$ is the semisimple component of a Levi decomposition of $\bar{L}$. Further, $R$ is nilpotent and $\operatorname{Cen}(R)=h$.

Proof. We first prove that $R$ is a nilpotent ideal of $\bar{L}$ and Cen $(R)=h$. To begin with, it is clear that $R$ is an ideal of $\bar{L}$ by Lemma 6.1. Now, if $h=1$, then $R=L e_{1,2}$. Hence $Z^{0}(R)=L e_{1,2}$ and $Z^{1}(R)=\left[L e_{1,2}, L e_{1,2}\right]=0$; and so Cen $(R)=1$. We now assume that $R$ is nilpotent and Cen $(R)=h$ when $h=m$ and prove that $R$ is nilpotent with Cen $(R)=m+1$ when $h=m+1$. Recall $N=2^{h}$ and $M=2^{h-1}=2^{m}$; and let $L_{1}=\sum_{i=1}^{M} L e_{i, M}$ and $R_{1}=\sum_{i=1}^{M-1} L e_{i, M}$. Then

$$
R=\left(L_{1}, R_{1}\right)=\left\{\left(l_{1}, r_{1}\right) \mid l_{1} \in L_{1} \text {, and } r_{1} \in R_{1}\right\}
$$


Now observe by Lemma 6.1 that if $I_{1}$ and $I_{2}$ are arbitrary subsets of $\{1, \ldots, M-1\}$ then

$$
\left[\sum_{i \in I_{1}} L e_{i, M}, \sum_{i \in I_{2}} L e_{i, M}\right]=\sum_{i \in I_{3}} L e_{i, M}
$$

where $I_{3}$ is also a subset of $\{1, \ldots, M-1\}$. Thus $Z^{j}\left(R_{1}\right)$ is an ideal of the form $\sum_{i \in I_{3}} L e_{i, M}$ for all $j$. Further, by Lemma 6.2,

$$
\left[L e_{M, M}, \sum_{i \in I_{1}} L e_{i, M}\right]=\sum_{i \in l_{1}} L e_{i, M} \text {. }
$$

Hence, we see that if $\sum_{i \in I} L e_{i, M}$ is an ideal of $L_{1}$ for some subset $I$ of $\{1, \ldots, M-1\}$, then $\left[L_{1}, \sum_{i \in I} L e_{i, M}\right]=\sum_{i \in I} L e_{i, M}$. Consequently,

$$
\left[L_{1}, Z^{j}\left(R_{1}\right)\right]=Z^{j}\left(R_{1}\right), \quad j=0,1, \ldots, m .
$$

We are now in a position to show that $Z^{j}(R)=\left(Z^{j-1}\left(R_{1}\right), Z^{j}\left(R_{1}\right)\right)$ for all $j$. If $j=1$, then

$$
Z^{1}(R)=\left[\left(L_{1}, R_{1}\right),\left(L_{1}, R_{1}\right)\right]=\left(\left[L_{1}, R_{1}\right]+\left[R_{1}, L_{1}\right],\left[R_{1}, R_{1}\right]\right)=\left(R_{1}, Z^{1}\left(R_{1}\right)\right) .
$$

Hence we assume this formula true for $j=k \geqq 1$ and prove it true for $j=k+1$. Now,

$$
\begin{aligned}
Z^{k+1}(R) & =\left[R, Z^{k}(R)\right]=\left[\left(L_{1}, R_{1}\right),\left(Z^{k-1}\left(R_{1}\right), Z^{k}\left(R_{1}\right)\right)\right] \\
& =\left(\left[L_{1}, Z^{k}\left(R_{1}\right)\right]+\left[R_{1}, Z^{k-1}\left(R_{1}\right)\right],\left[R_{1}, Z^{k}\left(R_{1}\right)\right]\right) \\
& =\left(Z^{k}\left(R_{1}\right)+Z^{k}\left(R_{1}\right), Z^{k+1}\left(R_{1}\right)\right)=\left(Z^{k}\left(R_{1}\right), Z^{k+1}\left(R_{1}\right)\right) .
\end{aligned}
$$

Consequently, by induction on $j$ the formula is seen to be true.

Now, it is clear from this formula that if $R_{1}$ is nilpotent and Cen $\left(R_{1}\right)=m$, then $R$ is nilpotent and Cen $(R)=m+1$. Thus we have shown that $R$ is nilpotent and Cen $(R)=h$, when $\bar{L}=\Omega^{h}(L)$.

Concluding the proof of the theorem we observe that $\bar{L} / R \cong L e_{N, N} \cong L$ and $L$ is semisimple. It follows that $R$ is a maximal solvable (indeed, nilpotent) ideal of $\bar{L}$. Since $L e_{N, N}$ is a semisimple subalgebra of $\bar{L}$, then $\bar{L}=R \oplus L e_{N, N}$ is a Levi decomposition.

8. We now assume for the remainder of this paper that $L$ is a complex semisimple Lie algebra of dimension $n$ and rank $l$. We will call an element $x_{0} \in L$ nilpotent if ad $x_{0}$ is a nilpotent transformation on $L$. By a theorem of Jacobson and Morozov, [3, p. 983], if $x_{0} \in L$ is nilpotent, then there exists $h_{0}$ and $y_{0} \in L$ such that $\left[h_{0}, x_{0}\right]$ $=2 x_{0},\left[h_{0}, y_{0}\right]=-2 y_{0}$, and $\left[x_{0}, y_{0}\right]=h_{0}$. Let $T$ be the Lie subalgebra of $L$ generated by $\left\{h_{0}, x_{0}, y_{0}\right\}$; we see that $T$ is a complex simple three-dimensional Lie algebra. Hence $L$ can be decomposed as a direct sum of irreducible representations of $T$, under the action ad $w: L \rightarrow L, w \in T$, of dimensions $\lambda_{1}+1, \lambda_{2}+1, \ldots, \lambda_{r}+1$. Therefore, by the theory of representations of $T$ [4, Chapter IV, pp. 1-8], the centralizer $Z$ of $y_{0}$ in $L$ is of dimension $r$ with a basis $\left\{y_{1}, \ldots, y_{r}\right\}$ such that $\left[h_{0}, y_{i}\right]$ $=-\lambda_{i} y_{i}, i=1, \ldots, r$. Further, the range ad $x_{0}(L)$ of ad $x_{0}$ in $L$ is complementary 
to $Z$ in $L$; that is, $L=Z \oplus$ ad $x_{0}(L)$. We will say that $x_{0} \in L$ is a principal nilpotent element if ad $x_{0}$ is nilpotent and $r=l$ (in general, $r \geqq l$ ). By [3, pp. 993-1000], principal nilpotent elements exist in $L$.

9. Recall $\bar{L}=\Omega^{h}(L), h$ is a positive integer, $N=2^{h}, \bar{G}$ is a connected Lie group with Lie algebra equal to $\bar{L}$ and $G$ is a connected Lie subgroup of $\bar{G}$ with Lie algebra equal to $L$. For the remainder of this paper, we will fix $h$ and $N$ and let $e_{i}=e_{i, N}, i=1, \ldots, N$. We now define the following bilinear form on $\bar{L}$ :

$$
\left\{\sum_{i=1}^{N} a_{i} e_{i}, \sum_{i=1}^{N} b_{i} e_{i}\right\}=\sum_{i=1}^{N}\left\langle a_{i}, b_{i}\right\rangle
$$

where $a_{i}, b_{i} \in L, i=1, \ldots, N$, and $\langle$,$\rangle is the Killing form on L$. Since $\langle$,$\rangle is$ nondegenerate on $L$, then it is clear that $\{$,$\} is nondegenerate on L$. Thus we identify $\bar{L}^{*}$ with $\bar{L}$ by defining

$$
\left(\sum_{i=1}^{N} a_{i} e_{i}\right)^{*}\left(\sum_{i=1}^{N} b_{i} e_{i}\right)=\left\{\sum_{i=1}^{N} b_{i} e_{i}, \sum_{i=1}^{N} a_{i} e_{i}\right\}
$$

where $a_{i}$ and $b_{i} \in L, i=1, \ldots, N$. Further, we see that

$$
\operatorname{Ad}^{*}(g)\left(\sum_{i=1}^{N} a_{i} e_{i}\right)^{*}\left(\sum_{i=1}^{N} b_{i} e_{i}\right)=\left\{\operatorname{Ad}\left(g^{-1}\right) \sum_{i=1}^{N} b_{i} e_{i}, \sum_{i=1}^{N} a_{i} e_{i}\right\}
$$

where $g \in \bar{G}$. In the sequel, we will omit the "*" from elements of $\bar{L}^{*}$; it will be clear from the context whether the element in question is in $\bar{L}$ or $\bar{L}^{*}$. Moreover, we continue our study of $I(\bar{G}, \bar{L}, \mathrm{Ad})$ by considering instead the isomorphic ring $\operatorname{IF}\left(\bar{G}, \bar{L}^{*}, \mathrm{Ad}^{*}\right)$; see $\S 2$.

Let $x_{0} \in L$ be nilpotent and let $\left\{h_{0}, x_{0}, y_{0}\right\}, Z,\left\{y_{1}, \ldots, y_{r}\right\}, \lambda_{1}+1, \ldots, \lambda_{r}+1$ be as in $\S 8$. Set $H=N \cdot r$, let $\boldsymbol{u}=\left(u_{1}, \ldots, u_{H}\right) \in C^{H}$ and define

$$
x(u)=\sum_{i=1}^{N}\left(x_{0}+\sum_{j=1}^{r} u_{j+r(i-1)} y_{j}\right) e_{i} \in \bar{L}^{*} .
$$

Lemma 9.1. Let $\psi: \bar{G} \times C^{H} \rightarrow \bar{L}^{*}$ be defined by letting $\psi(g, u)=\operatorname{Ad}^{*}(g)(x(u))$ for $g \in \bar{G}$ and $\boldsymbol{u}=\left(u_{1}, \ldots, u_{H}\right) \in \boldsymbol{C}^{H}$. Then

$$
\begin{aligned}
d \psi_{(1, u)}\left(\sum_{k=1}^{N} a_{k} e_{k}, v\right) & \\
& =\sum_{k=1}^{N} \sum_{i=1}^{k} c_{k, i}\left[a_{k}, x_{0}+\sum_{j=1}^{r} u_{j+r(i-1)} y_{j}\right] e_{N-k+i}+\sum_{i=1}^{N}\left(\sum_{j=1}^{r} v_{j+r(i-1)} y_{j}\right) e_{i},
\end{aligned}
$$

where $1=$ identity of $\bar{G}, a_{1}, \ldots, a_{N} \in L, v=\left(v_{1}, \ldots, v_{H}\right) \in C^{H}, c_{k, i}=0$ or $1, c_{N, i}=c_{k, 1}$ $=c_{k, k}=1, k=1, \ldots, N, i=1, \ldots, k$, and $d \psi_{(1, u)}: \bar{L} \times C^{H} \rightarrow \bar{L}^{*}$ is the differential of $\psi$ evaluated at $(1, u) \in \bar{G} \times C^{H}$. Here we identify canonically the tangent space of the complex analytic manifold $G \times C^{H}$ at any point $(g, u) \in \bar{G} \times C^{H}$ with $\bar{L} \times C^{H}$, and identify canonically the tangent space of $\bar{L}^{*}$ at any point of it with $\bar{L}^{*}$ itself. 
Proof. Let $1 \leqq k \leqq N$ and we compute, for $a, b_{1}, \ldots, b_{N} \in L$,

$$
\begin{aligned}
d \psi_{(1, u)}\left(a e_{k}, \mathbf{0}\right)\left(\sum_{i=1}^{N} b_{i} e_{i}\right) & =\frac{d}{d t}\left(\psi\left(\exp \operatorname{tae}_{k}, u\right)\left(\sum_{i=1}^{N} b_{i} e_{i}\right)\right)_{t=0} \\
& =\frac{d}{d t}\left(\operatorname{Ad}^{*}\left(\exp \operatorname{tae}_{k}\right)(x(u))\left(\sum_{i=1}^{N} b_{i} e_{i}\right)\right)_{t=0} \\
& =\frac{d}{d t}\left\{\operatorname{Ad}\left(\exp -\operatorname{tae}_{k}\right)\left(\sum_{i=1}^{N} b_{i} e_{i}\right), x(u)\right\}_{t=0} \\
& =\left\{\sum_{i=1}^{k}-c_{k, i}\left[a, b_{N-k+i}\right] e_{i}, \sum_{i=1}^{N}\left(x_{0}+\sum_{j=1}^{r} u_{j+r(i-1)} y_{j}\right) e_{i}\right\} \\
& =\sum_{i=1}^{k} c_{k, i}\left\langle-\left[a, b_{N-k+i}\right], x_{0}+\sum_{j=1}^{r} u_{j+r(i-1)} y_{j}\right\rangle \\
& =\sum_{i=1}^{k} c_{k, i}\left\langle b_{N-k+i},\left[a, x_{0}+\sum_{j=1}^{r} u_{j+r(i-1)} y_{j}\right]\right\rangle \\
& =\left\{\sum_{i=1}^{k} b_{N-k+i} e_{N-k+i}, \sum_{i=1}^{k} c_{k, i}\left[a, x_{0}+\sum_{j=1}^{r} u_{j+r(i-1)} y_{j}\right] e_{N-k+1}\right\} \\
& =\left\{\sum_{i=1}^{N} b_{i} e_{i}, \sum_{i=1}^{k} c_{k, i}\left[a, x_{0}+\sum_{j=1}^{r} u_{j+r(i-1)} y_{j}\right] e_{N-k+i}\right\} .
\end{aligned}
$$

Hence,

$$
d \psi_{(1, u)}\left(a e_{k}, \mathbf{0}\right)=\sum_{i=1}^{k} c_{k, i}\left[a, x_{0}+\sum_{j=1}^{r} u_{j+r(i-1)} y_{j}\right] e_{N-k+i}
$$

where $c_{k, i}=0$ or $1, c_{N, i}=c_{k, 1}=c_{k, k}=1, k=1, \ldots, N, i=1, \ldots, k$, by Lemma 6.1 and Lemma 6.2.

Finally, we compute for $v=\left(v_{1}, \ldots, v_{H}\right) \in C^{H}$,

$$
\begin{aligned}
d \psi_{(1, u)}(0, v)\left(\sum_{i=1}^{N} b_{i} e_{i}\right) & =\frac{d}{d t}\left(\psi(1, u+t v)\left(\sum_{i=1}^{N} b_{i} e_{i}\right)\right)_{t=0} \\
& =\frac{d}{d t}\left(\operatorname{Ad}^{*}(1) x(u+t v)\left(\sum_{i=1}^{N} b_{i} e_{i}\right)\right)_{t=0} \\
& =\frac{d}{d t}\left\{\sum_{i=1}^{N} b_{i} e_{i}, \sum_{i=1}^{N}\left(x_{0}+\sum_{j=1}^{r}\left(u_{j+r(i-1)}+t v_{j+r(i-1)}\right) y_{j}\right) e_{i}\right\}_{t=0} \\
& =\left\{\sum_{i=1}^{N} b_{i} e_{i}, \sum_{i=1}^{N}\left(\sum_{j=1}^{r} v_{j+r(i-1)} y_{j}\right) e_{i}\right\}
\end{aligned}
$$

Hence,

$$
d \psi_{(1, u)}(0, v)=\sum_{i=1}^{N}\left(\sum_{j=1}^{r} v_{j+r(i-1)} y_{j}\right) e_{i}
$$

The lemma clearly follows from these two computations.

Now let $f$ be any complex-valued function defined on an open subset $U$ of $\bar{L}^{*}$ containing $\sum_{i=1}^{N} x_{0} e_{i}$. Then we let $\tilde{f}^{2}$ be the following function defined on an open neighborhood of the origin in $C^{H}$ :

$$
\tilde{f}(\boldsymbol{u})=f(x(u)), \quad \text { where } u=\left(u_{1}, \ldots, u_{H}\right) \in C^{H}, \text { and } x(u) \in U .
$$


THEOREM 9.2. There exists an open set $W$ of $C^{H}$ containing the origin such that

$$
\Lambda(W)=\left\{\operatorname{Ad}^{*}(g)(x(\boldsymbol{u})) \mid g \in \bar{G} \text { and } \boldsymbol{u} \in W\right\}
$$

is an open subset of $\bar{L}^{*}$. Furthermore, the mapping $p \rightarrow \tilde{p}$ is an injective algebra homomorphism of $\operatorname{IF}\left(\bar{G}, \bar{L}^{*}, \mathrm{Ad}^{*}\right)$ into the algebra of polynomial functions on $W$.

Proof. Define $\psi: \bar{G} \times C^{H} \rightarrow \bar{L}^{*}$ as in Lemma 9.1. Then

$$
d \psi_{(1,0)}\left(\sum_{i=1}^{N} a_{i} e_{i}, v\right)=\sum_{k=1}^{N} \sum_{i=1}^{k} c_{k, i}\left[a_{k}, x_{0}\right] e_{N-k+i}+\sum_{i=1}^{N}\left(\sum_{j=1}^{r} v_{j+r(i-1)} y_{j}\right) e_{i} .
$$

We want to show that $d \psi_{(1,0)}$ is surjective. First, we observe that by letting $a_{2}=a_{3}=\cdots=a_{N}=0, \boldsymbol{v}=\mathbf{0}$ and $a_{1}$ vary over $L$, then $d \psi_{(1,0)}\left(\bar{L} \times C^{H}\right)$ contains ad $x_{0}(L) e_{N}$. So let us assume that $d \psi_{(1,0)}\left(\bar{L} \times C^{H}\right)$ contains $\sum_{i=1}^{q}$ ad $x_{0}(L) e_{N+1-i}$, for $1 \leqq q<N$, then we will show that $d \psi_{(1,0)}\left(\bar{L} \times C^{H}\right)$ contains $\sum_{i=1}^{q+1}$ ad $x_{0}(L) e_{N+1-i}$. For this we let $a_{i}=0, i=1, \ldots, N$ and $i \neq q+1, \boldsymbol{v}=\mathbf{0}$ and let $a_{q+1}$ vary over $L$. Then since $c_{q+1,1}=1$, we see that $d \psi_{(1,0)}\left(L \times C^{H}\right)$ contains the set of vectors

$$
\left\{\left[a_{q+1}, x_{0}\right] e_{(N+1)-(q+1)}+\sum_{i=2}^{q+1} c_{q+1, i}\left[a_{q+1}, x_{0}\right] e_{N-(q+1)+i} \mid a_{q+1} \in L\right\} .
$$

But $d \psi_{(1,0)}\left(\bar{L} \times C^{H}\right)$ is a vector space and it already contains $\sum_{i=1}^{q}$ ad $x_{0}(L) e_{N+1-i}$. Hence, it contains ad $x_{0}(L) e_{N+1-(q+1)}$ and thus $\sum_{i=1}^{q+1}$ ad $x_{0}(L) e_{N+1-i}$. Therefore, by induction we see that $d \psi_{(1,0)}\left(\bar{L} \times C^{H}\right)$ contains $\sum_{i=1}^{N}$ ad $x_{0}(L) e_{i}$. Finally, using the notation of $\S 8$, we have $Z \oplus$ ad $x_{0}(L)=L$. Thus by letting $a_{1}=\cdots=a_{N}=0$ and letting $v$ vary over $C^{H}$, we see that $d \psi_{(1,0)}\left(\bar{L} \times C^{H}\right)$ contains $\sum_{i=1}^{N} Z e_{i}$ and hence $d \psi_{(1,0)}\left(\bar{L} \times C^{H}\right)=\bar{L}^{*}$.

Now since $d \psi$ is surjective at $(1, \mathbf{0})$, there exists an open set $W \subset C^{H}$ with $\mathbf{0} \in W$ and such that $d \psi_{(1, u)}$ is surjective for all $\boldsymbol{u} \in W$. Hence $d \psi_{(g, u)}=\operatorname{Ad}^{*}(g) d \psi_{(1, u)}$ is surjective for all $g \in \bar{G}, \boldsymbol{u} \in W$. Therefore, it follows from the theory of analytic manifolds that $\psi(\bar{G} \times W)=\Lambda(W)$ is open in $\bar{L}^{*}$.

The second statement follows directly. For let $p \in \operatorname{IF}\left(\bar{G}, \bar{L}^{*}, \mathrm{Ad}^{*}\right)$ and let $\tilde{p}(u)=0$ for all $\boldsymbol{u} \in W$. Then $p\left(\operatorname{Ad}^{*}(g) x(\boldsymbol{u})\right)=p(x(\boldsymbol{u}))=\tilde{p}(\boldsymbol{u})=0$ for all $\boldsymbol{u} \in W$ and $g \in \bar{G}$. Thus $p$ is zero on $\Lambda(W)$; and as $\Lambda(W)$ is open in $\bar{L}^{*}, p=0$ everywhere in $\bar{L}^{*}$. Consequently, the map $p \rightarrow \tilde{p}$ is injective. Since it is clearly an algebra homomorphism, we are done.

For the remainder of this paper, if $p \in I F\left(\bar{G}, \bar{L}^{*}, \mathrm{Ad}^{*}\right)$ then $\tilde{p}$ will denote the previously defined function with domain $W$.

Now by the theorem of Chevalley in $\S 1, I(G, L, \mathrm{Ad})$ is generated by $l$ algebraically independent homogeneous polynomials of distinct degrees $d_{1}, \ldots, d_{s}$; assume $n_{i}$ of them are of degree $d_{i}, i=1, \ldots, s$. Then by Theorem 5.1 and the fact that $I(\bar{G}, \bar{L}, \mathrm{Ad})$ is isomorphic to $I F\left(\bar{G}, \bar{L}^{*}, \mathrm{Ad}^{*}\right)(\S 2), I\left(\bar{G}, \bar{L}^{*}, \mathrm{Ad}^{*}\right)$ has at least $N l$ algebraically independent homogeneous polynomial functions, say $p_{1}, \ldots, p_{N l}$, where $N n_{i}$ of these are of degree $d_{i}, i=1, \ldots, s$. 
Corollary 9.3. Let $p_{1}, \ldots, p_{N l} \in I F\left(\bar{G}, \bar{L}^{*}, \mathrm{Ad}^{*}\right)$ be as above. Then $\tilde{p}_{1}, \ldots, \tilde{p}_{N l}$ are polynomial functions defined on $W$ which are algebraically independent.

Proof. Let $Q$ be a complex polynomial in $N l$ variables such that $Q\left(\tilde{p}_{1}, \ldots, \tilde{p}_{N l}\right)$ $=0$. Then $Q\left(p_{1}, \ldots, p_{N l}\right)=0$ by Theorem 9.2. Hence $Q=0$ since $p_{1}, \ldots, p_{N l}$ are algebraically independent.

10. Now we define a vector field $E$ on $\bar{L}^{*}$ by setting

$$
E f(y)=y f(y)=(d / d t)\{f(y+t y)\}_{t=0},
$$

where $y \in \bar{L}^{*}$ and $f$ is any holomorphic function defined on some neighborhood of $y$.

THEOREM 10.1. Let $W$ be as in Theorem 9.2. Then there exists a differential operator $\tilde{E}$ on $W$ such that $E f(x(\boldsymbol{u}))=\tilde{E} \tilde{f}(\boldsymbol{u})$, where $\boldsymbol{u}=\left(u_{1}, \ldots, u_{H}\right) \in W$ and where $f$ is any holomorphic function on $\Lambda(W)$ such that $f\left(\operatorname{Ad}^{*}(g) x(\boldsymbol{u})\right)=f(x(\boldsymbol{u}))$ for all $\boldsymbol{u} \in W$ and $g \in \bar{G}$.

Further, if we define $\lambda_{j+r(i-1)}=\lambda_{j}, j=1, \ldots, r, i=1, \ldots, N$, then

$$
\tilde{E}=\sum_{j=1}^{H}\left(1+\lambda_{j} / 2\right) u_{j} \frac{\partial}{\partial u_{j}}
$$

Proof. Let $f$ be any holomorphic function defined on $\Lambda(W)$. Then $f$ defines a function $f^{\psi}(g, u)=f\left(\operatorname{Ad}^{*}(g) x(u)\right)$ for $g \in \bar{G}$ and $\boldsymbol{u} \in W$. Now let $\boldsymbol{u} \in W$ and define $\boldsymbol{v}=\left(v_{1}, \ldots, v_{H}\right) \in \boldsymbol{C}^{H}$ by letting $v_{j+r(i-1)}=\left(1+\lambda_{j} / 2\right) u_{j+r(i-1)}, j=1, \ldots, r$ and $i=1, \ldots, N$. Then we have, by $\S 8$ and Lemma 9.1,

$$
\begin{aligned}
& d \psi_{(1, u)}\left(\frac{1}{2} h_{0} e_{N}, v\right) \\
& =\sum_{i=1}^{N}\left[\frac{1}{2} h_{0}, x_{0}+\sum_{j=1}^{r} u_{j+r(i-1)} y_{j}\right] e_{i}+\sum_{i=1}^{N}\left(\sum_{j=1}^{r}\left(1+\lambda_{j} / 2\right) u_{j+r(i-1)} y_{j}\right) e_{i} \\
& =\sum_{i=1}^{N}\left(x_{0}+\sum_{j=1}^{r} u_{j+r(i-1)}\left(-\lambda_{j} / 2\right) y_{j}\right) e_{i}+\sum_{i=1}^{N}\left(\sum_{j=1}^{r}\left(1+\lambda_{j} / 2\right) u_{j+r(i-1)} y_{j}\right) e_{i}=x(\boldsymbol{u}) .
\end{aligned}
$$

Therefore, we have

$$
\begin{aligned}
\left(\left(\frac{1}{2} h_{0} e_{N}, \boldsymbol{v}\right) f^{\psi}\right)(1, \boldsymbol{u}) & =\left(\frac{1}{2} h_{0} e_{N}, \boldsymbol{v}\right) f \circ \psi(1, \boldsymbol{u}) \\
& =\left(d \psi_{(1, u)}\left(\frac{1}{2} h_{0} e_{N}, \boldsymbol{v}\right) f\right)(x(\boldsymbol{u}))=(x(\boldsymbol{u}) f)(x(\boldsymbol{u}))=E f(x(\boldsymbol{u})) .
\end{aligned}
$$

Now $f\left(\operatorname{Ad}^{*}(g) x(\boldsymbol{u})\right)=f(x(\boldsymbol{u}))$ for all $\boldsymbol{u} \in W$ and $g \in \bar{G}$; hence $f^{\psi}(g, \boldsymbol{u})=\tilde{f}(\boldsymbol{u})$ for all $g \in \bar{G}$. Therefore,

$$
\begin{aligned}
E f(x(\boldsymbol{u})) & =\left(\left(\frac{1}{2} h_{0} e_{N}, \boldsymbol{v}\right) f^{\psi}\right)(1, \boldsymbol{u})=\boldsymbol{v} \tilde{f}(\boldsymbol{u}) \\
& =\left(\left(\sum_{j=1}^{H}\left(1+\lambda_{j} / 2\right) u_{j} \frac{\partial}{\partial u_{j}}\right) \tilde{f}\right)(\boldsymbol{u}) .
\end{aligned}
$$

Consequently, $\tilde{E}=\sum_{j=1}^{H}\left(1+\lambda_{j} / 2\right) u_{j} \partial / \partial u_{j}$ will satisfy the theorem.

11. We now let $x_{0}$ be a principal nilpotent element of $L$. Then $r=l$ and $H=N l$. Further, assume that $q_{1}, \ldots, q_{l}$ are the algebraically independent homogeneous 
generators of $I(G, L, \mathrm{Ad})$. Then it is known, see [3] or [6, Theorem 1, p. 312], that the degree of $q_{i}=1+\lambda_{i} / 2, i=1, \ldots, l$, after a suitable reordering of the set $\left\{q_{1}, \ldots, q_{l}\right\}$. Consequently, after a suitable reordering of $\left\{p_{1}, \ldots, p_{H}\right\}$, we have

the degree of $p_{i}=1+\lambda_{i} / 2, \quad i=1, \ldots, H$.

THEOREM 11.1. Let $W$ be as in Theorem 9.2. Then the map $p \rightarrow \tilde{p}$ is an algebra isomorphism of $\operatorname{IF}\left(\bar{G}, \bar{L}^{*}, \mathrm{Ad}^{*}\right)$ onto the algebra of all polynomial functions on $W$.

Proof. By Theorem 9.2, we need only show that the map $p \rightarrow \tilde{p}$ is surjective. So let $J$ be the algebra of all polynomial functions on $W$. Further, let $\tilde{I}$ be the subalgebra of $J$ generated by the set $\left\{\tilde{p}_{1}, \ldots, \tilde{p}_{H}\right\}$. Finally, let $I$ be the subalgebra of $\operatorname{IF}\left(\bar{G}, \bar{L}^{*}, \mathrm{Ad}^{*}\right)$ generated by the set $\left\{p_{1}, \ldots, p_{H}\right\}$.

Let us now make the following observation. If $D\left(n_{1}, \ldots, n_{H}\right)$ is equal to the monomial function $u_{1}^{n}{ }_{1} \cdots u_{H}^{n}$ on $W$, then

$$
\widetilde{E}\left(D\left(n_{1}, \ldots, n_{H}\right)\right)=\left(\sum_{i=1}^{H}\left(1+\lambda_{i} / 2\right) n_{i}\right) D\left(n_{1}, \ldots, n_{H}\right) .
$$

Therefore, if $p \in J$ is such that $\tilde{E} p=j p$, then $p$ must be a linear combination of monomials $D\left(n_{1}, \ldots, n_{H}\right)$ for which $\sum_{i=1}^{H}\left(1+\lambda_{i} / 2\right) n_{i}=j$. Consequently, it is clear that $\sum_{j=0}^{\infty}(\operatorname{dim}\{p \in J \mid \widetilde{E} p=j p\}) T^{j}$ is a formal power series represented by $\left(\left(1-T^{1+\lambda_{1} / 2}\right) \cdots\left(1-T^{1+\lambda_{H} / 2}\right)\right)^{-1}$ where $\operatorname{dim}\{p \in J \mid \tilde{E} p=j p\}$ is the dimension of $\{p \in J \mid \widetilde{E} p=j p\}$ as a complex vector space.

On the other hand, since the map $p \rightarrow \tilde{p}$ is injective and since $E p(x(u))=\tilde{E} \tilde{p}(\boldsymbol{u})$ for $p \in \operatorname{IF}\left(\bar{G}, \bar{L}^{*}, \mathrm{Ad}^{*}\right)$ and $u \in W$, then

$$
\sum_{j=0}^{\infty}(\operatorname{dim}\{p \in \tilde{I} \mid \tilde{E} p=j p\}) T^{j}=\sum_{j=0}^{\infty}(\operatorname{dim}\{p \in I \mid E p=j p\}) T^{j} .
$$

But this latter series is also represented by $\left(\left(1-T^{1+\lambda_{1} / 2}\right) \cdots\left(1-T^{1+\lambda_{H} / 2}\right)\right)^{-1}$, since the degree of $p_{i}$ is $1+\lambda_{i} / 2, i=1, \ldots, H$.

Therefore,

$$
\operatorname{dim}\{p \in J \mid \widetilde{E} p=j p\}=\operatorname{dim}\{p \in \tilde{I} \mid \tilde{E} p=j p\} \quad \text { for all } j .
$$

Now, since $\tilde{I} \subseteq J$, then $\{p \in J \mid \tilde{E} p=j p\}=\{p \in \tilde{I} \mid \tilde{E} p=j p\}$ for all $j$.

Since $J=\sum_{j=0}^{\infty}\{p \in J \mid \tilde{E} p=j p\}$, it follows that $\tilde{I}=J$, and we are done.

CoROLlaRY 11.1. IF $\left(\bar{G}, \bar{L}^{*}, \mathrm{Ad}^{*}\right)$ is generated by the $H$ algebraically independent homogeneous polynomials $p_{1}, \ldots, p_{H}, H=N l$.

Proof. Let $p \in \operatorname{IF}\left(\bar{G}, \bar{L}^{*}, \mathrm{Ad}^{*}\right)$ and let $W$ be as in Theorem 9.2. Then $\tilde{p}$ is a polynomial function on $W$, say $\tilde{p}=f\left(u_{1}, \ldots, u_{H}\right)$, where $f$ is a polynomial in $H$ variables. Now by Theorem 11.1 , there exists polynomials $f_{1}, \ldots, f_{H}$ each in $H$ variables such that $u_{i}=f_{i}\left(\tilde{p}_{1}, \ldots, \tilde{p}_{H}\right), i=1, \ldots, H$. Consequently,

$$
\begin{aligned}
\tilde{p} & =f\left(u_{1}, \ldots, u_{H}\right) \\
& =f\left(f_{1}\left(\tilde{p}_{1}, \ldots, \tilde{p}_{H}\right), \ldots, f_{H}\left(\tilde{p}_{1}, \ldots, \tilde{p}_{H}\right)\right)=f_{0}\left(\tilde{p}_{1}, \ldots, \tilde{p}_{H}\right),
\end{aligned}
$$


where $f_{0}$ is a polynomial in $H$ variables. Therefore, as the map $p \rightarrow \tilde{p}$ is an algebra isomorphism, $p=f_{0}\left(p_{1}, \ldots, p_{H}\right)$. And so we see that $p_{1}, \ldots, p_{H}$ generate $\operatorname{IF}\left(\bar{G}, \bar{L}^{*}, \mathrm{Ad}^{*}\right)$.

COROLlaRy 11.3. I $\left(\bar{G}, \bar{L}\right.$, Ad) is generated by the $\left(2^{h}\right) l$ algebraically independent homogeneous polynomials which are determined, as in Theorem 4.1 and Theorem 5.1, by the l generators of $I(G, L, \mathrm{Ad})$.

Proof. This corollary is a direct consequence of Corollary 11.2 and the fact that $I(\bar{G}, \bar{L}, \mathrm{Ad})$ is isomorphic to $I F\left(\bar{G}, \bar{L}^{*}, \mathrm{Ad}^{*}\right)$.

\section{REFERENCES}

1. C. Chevalley, Invariants of finite groups generated by reflections, Amer. J. Math. 77 (1955), 778-782. MR 17, 345; 1436.

2. N. Jacobson, Lie algebras, Interscience Tracts in Pure and Appl. Math., no. 10, Interscience, New York, 1962. MR 26 \#1345.

3. B. Kostant, The principal three-dimensional subgroup and the Betti numbers of a complex simple Lie group, Amer. J. Math. 81 (1959), 973-1032. MR 22 \#5693.

4. J. P. Serre, Algèbres de Lie semi-simples complexes, Benjamin, New York, 1966. MR 35 \#6721.

5. V. S. Varadarajan, Lie groups, University of California, Los Angeles, 1967 (mimeographed notes).

6. - On the ring of invariant polynomials on a semisimple Lie algebra, Amer. J. Math. 90 (1968), 308-317. MR 37 \#1529.

Michigan State University,

East Lansing, Michigan 48823 